\title{
Protective effect of donepezil hydrochloride on cerebral ischemia/reperfusion injury in mice
}

\author{
TIANJUN WANG, PEIYUAN LV, WEI JIN, HEZHEN ZHANG, JINGFANG LANG and MINGYUE FAN \\ Department of Neurology, Hebei General Hospital, Shijiazhuang, Hebei 050051, P.R. China
}

Received September 9, 2013; Accepted November 8, 2013

DOI: $10.3892 / \mathrm{mmr} .2013 .1823$

\begin{abstract}
The aim of this study was to investigate the effects of donepezil hydrochloride $(\mathrm{DH})$ on the expression of the calpain I-cyclin-dependent kinase5/p25 (CDK5/p25) pathway in the hippocampal CA1 region in mice with cerebral ischemiareperfusion (I/R). Mice were randomly divided and assigned to the sham operation group (SO), the model group (MG) and the DH treatment group (TG). The pathological appearance of the hippocampal CA1 region and the expression of calpain I and CDK5/p25, were observed on the 4th, 6th and 8th week of the I/R surgery. Within the same time periods, superoxide dismutase (SOD) activity and malondialdehyde (MDA) content were also determined. At each postoperative time point, the normal neuron count in the hippocampal CA1 region in the $\mathrm{MG}$ was significantly lower than that in the SO $(\mathrm{P}<0.05)$, whereas the calpain I and CDK5/p25 expression, SOD activity and MDA content in the MG were significantly higher than those in the SO $(\mathrm{P}<0.05)$. The normal neuron count of the hippocampal CA1 region in the TG increased significantly $(\mathrm{P}<0.05)$, whereas the calpain I and CDK5/p25 expression, SOD activity and MDA content in the TG were significantly lower than those in the MG $(\mathrm{P}<0.05)$. $\mathrm{DH}$ has protective effects against ischemic damage. The ability of DH to improve learning and memory in mice may be due to its ability to decrease the expression of the calpain I-CDK5/p25 pathway and reduce oxidative damage.
\end{abstract}

\section{Introduction}

Transient prosencephalic ischemia-reperfusion (I/R) injury is able to cause neuronal death in the hippocampal CA1 region, thus resulting in damage to learning and memory, however, its exact pathogenesis remains unclear (1). Factors that contribute to cerebral I/R injury include oxidative stress caused by the production of reactive oxygen species (ROS), disruption of

Correspondence to: Dr Peiyuan Lv, Department of Neurology, Hebei General Hospital, no. 348 Hepingxi Road, Shijiazhuang, Hebei 050051, P.R. China

E-mail: peiyuanlven@163.com

Key words: ischemia-reperfusion, memory, donepezil hydrochloride, calpain I, cyclin-dependent kinase 5, oxidative stress
$\mathrm{Ca}^{2+}$ homeostasis, activation of proteases and excitotoxicity of glutamatergic neurons (1-4). Among these factors, increased ROS production and cytosolic free $\mathrm{Ca}^{2+}$ overload are major contributors to I/R-induced injury $(2,3,5)$. Increases in neuronal cytosolic $\mathrm{Ca}^{2+}$ levels have been observed during ischemia and reperfusion $(3,5)$. One function of cytosolic $\mathrm{Ca}^{2+}$ in the pathogenesis of I/R-induced cerebral injury has been hypothesized to be the activation of the $\mathrm{Ca}^{2+}$-dependent protease calpain (6). Calpain exists in myocytes in two primary isoforms, namely, micro (calpain I) and milli (calpain II), which are named based on the respective amounts of $\mathrm{Ca}^{2+}$ required for their activation in vitro. When whole or regional cerebral ischemia occurs, inner neuronal $\mathrm{Ca}^{2+}$ is overloaded and calpain generation increases, which in turn causes cell damage (6). The downstream factor of calpain, cyclin-dependent kinase 5 (CDK5), is a multifaceted serine/threonine kinase protein that has important functions in the nervous system. Over the past decade, CDK5 activity has been demonstrated to regulate numerous events during brain development, including neuronal migration, as well as during axon and dendrite development. Recent evidence also suggests that CDK5 is pivotal in synaptic plasticity, behavior and cognition (7). Under pathological conditions, p35 may be truncated into p25, which is able to markedly and consistently activate CDK5, change the cellular localization of CDK5 and ultimately cause neuronal death (8). CDK5 dysfunction has been implicated in a number of neurological disorders and neurodegenerative diseases, including Alzheimer's disease (AD), amyotrophic lateral sclerosis, Niemann-Pick type $\mathrm{C}$ disease and ischemia $(7,8)$. CDK 5 hyperactivation, which is caused by the conversion of p35 to p25 by calpain during neurotoxicity, also contributes to the pathological state, however, its role in cognitive impairment following I/R injury remains unclear.

ROS-mediated oxidative insults during cerebral I/R injury may damage the vulnerable regions of the brain (9). Certain brain regions, including the cortex and the hippocampus, are more vulnerable to ischemia than other regions. Severe damage to the hippocampus results in difficulties in forming new memories (10). Repeated cerebral ischemia may lead to the accumulation of certain cellular active substances in the body and thus demonstrate a neurotoxic effect. This phenomenon may be an important factor in the occurrence of cognitive impairment (11). Oxidative damage caused by free radicals also involves mitochondrial damage, cell necrosis and apoptotic processes. Superoxide dismutase (SOD) and malondialdehyde (MDA) are important indicators in evaluating the 
extent of oxidation and antioxidation (12). Recent studies have linked I/R-induced ROS production to the oxidative modification of $\mathrm{Ca}^{2+}$ handling proteins (13). Therefore, the production of ROS and the overexpression of the calpain/CDK5 pathway are involved in the pathogenesis of I/R-induced cognitive impairment.

Donepezil ( $\pm-2-(1-$ benzylpiperidin-4-yl) methyl-5,6-dimethoxy-indan-1-one monohydrochloride) is a potent acetylcholinesterase inhibitor that has been previously demonstrated to be effective in improving cognition in patients with AD (14). In addition, pretreatment with donepezil hydrochloride $(\mathrm{DH})$ prior to the induction of focal cerebral ischemia has been demonstrated to significantly attenuate cerebral infarction volume (15). DH may also protect rat cortical neurons from glutamatergic neurotoxicity (16). These studies imply that DH may provide benefits for the treatment of ischemia. Recent studies have demonstrated that DH has a neuroprotective effect and this effect does not involve anti-cholinesterase (17). DH is able to inhibit the fast inflow of $\mathrm{Na}^{+}$and $\mathrm{Ca}^{2+}$, reduce the release of glutamate and therefore oppose the neurotoxicity induced by depolarization (18). However, few studies have investigated the effect of DH treatment on prosencephalic ischemia and the involvement of the calpain I/CDK5 pathway in the ameliorating effect of DH on learning and memory.

In the present study, the therapeutic effects of DH on prosencephalic ischemia in mice, including its effect on spatial memory function, are determined through histopathological evaluation. Calpain I and CDK5/p25 expression, SOD activity and MDA content are also analyzed in the ischemic mouse hippocampus.

\section{Materials and methods}

Animals and grouping. A total of 250 three-month old male mice were provided by the Experimental Animal Center of Hebei Medical University (Shijiazhuang, Hebei, China). The mice weighed $32.3 \pm 2.4 \mathrm{~g}$ and were bred in natural light. The circadian ratio was $12 \mathrm{~h} / 12 \mathrm{~h}$ at an ambient temperature of 20 to $25^{\circ} \mathrm{C}$. The mice were provided with food and water within the 1 week acclimatization period. The mice were then randomly divided into three groups: the sham operation group (SO, n=70), the model group $(\mathrm{MG}, \mathrm{n}=90)$ and the treatment group (TG, $\mathrm{n}=90)$. The present study was performed in strict accordance with the recommendations in the Guide for the Care and Use of Laboratory Animals of the National Institutes of Health (Bethesda, MD, USA). The animal use protocol has been reviewed and approved by the Institutional Animal Care and Use Committee (IACUC) of the Hebei General Hospital (Shijiazhuang, Hebei, China).

Animal model preparation. The following methods are based on literature with slight modifications (7). The mice were anesthetized with an intraperitoneal injection of $10 \%$ chloral hydrate $(0.035 \mathrm{ml} / 10 \mathrm{~g})$. Next, the bilateral common carotid artery was separated. The no. 4-0 silk thread was used to obstruct blood flow for $30 \mathrm{~min}$. Simultaneously, the tail was cut at $\sim 1 \mathrm{~cm}$ from the tail tip for $\sim 0.3 \mathrm{ml}$ of bloodletting. Once the blood flow was restored for $10 \mathrm{~min}$, the blood flow was obstructed again for $30 \mathrm{~min}$. This operation was repeated three times. Following the third blood flow reperfusion, careful observation was conducted for 30 min prior to the skin being sutured. The SO was only performed by isolating the bilateral common carotid artery and by thread binding without obstructing blood flow and tail bleeding. On the second day postoperative, the TG was orally administered $3 \mathrm{mg} / \mathrm{kg} /$ day of DH (Eisai Pharmaceutical Co., Ltd., Tokyo, Japan), whereas the SO and the MG were administered the same volume of saline.

Achievement tests of mouse learning and memory. The mouse water maze automatic recorder, manufactured by the Institute of Materia Medica, Chinese Academy of Medical Sciences (no. 3850; Beijing, China), is a brown Plexiglass slot $1.1 \mathrm{~m}$ in length, $0.7 \mathrm{~m}$ in width and $0.4 \mathrm{~m}$ in height. The ladder at one end of the slot is only able to be reached in one way. The mouse may climb up the ladder and rest. The inner slot was mazelike with multiple blind ends to prevent the mouse from seeing the ladder. Numerous automatic sensing devices automatically recorded the following data for $3 \mathrm{~min}$ : water depth, $10 \mathrm{~cm}$ and water temperature, $22 \pm 1^{\circ} \mathrm{C}$. Prior to the test, the mouse was trained to swim through the maze. Swimming time was recorded as the duration of time it took for the mouse to swim the entire distance; swimming time $>3$ min was recorded as $3 \mathrm{~min}$. On the 29th day (4th week), 43rd day (6th week) and 57th day (8th week) postoperative, the test achievements were recorded as learning scores and the achievements on the 30th day (4th week), 44th day (6th week) and 58th day (8th week) postoperative were recorded as memory scores.

Morphological observation. Six mice from each group were subjected to cardio-perfusion fixation. The section between the optic chiasm and the mammillary bodies was obtained and a coronal slice with a thickness of $6 \mu \mathrm{m}$ was performed. The slice was stained with hematoxylin for $5 \mathrm{~min}$ and $1 \%$ eosin for $4 \mathrm{~min}$. Two slices from the same parts of each mouse were selected and six non-overlapping regions in the hippocampal CA1 region of each slice were selected at a range of $0.1 \mathrm{~mm}$ for the regional normal neuron count and the sum from all the regions. The round or oval nucleolus without cell shrinkage and edema was counted as normal.

Immunohistochemical staining. Six mice from each group were subjected to cardio-perfusion fixation. The brain tissue between the optic chiasm and the mammillary bodies was obtained and subjected to external fixation in $4 \%$ paraformaldehyde solution for $24 \mathrm{~h}$. Continuous coronal slices at a thickness of $\sim 5 \mu \mathrm{m}$ were then performed. The operation strictly followed the manufacturer's instructions (purchased from Wuhan Boster Biological Technology, Ltd., Wuhan, Hubei, China). Each slice was counted in 10 fields and the amount of calpain I antigen was measured using the average optical density (OD) value of the positive cell staining.

Western blot. Six mice from each group were anesthetized and subsequently decapitated. The bilateral hippocampus was isolated on ice and then lysed according to the manufacturer's instructions. Protein quantification was performed using the bicinchoninic acid method. The products were then isolated by $15 \%$ SDS-polyacrylamide gel electrophoresis, transferred onto a PVDF membrane and blocked with the blocking solution at room temperature for $1 \mathrm{~h}$. The monoclonal primary antibody diluted with the blocking solution ( $\beta$-actin, 1:1,000) 
was then added, followed by the addition of polyclonal antibody (CDK5, C8, 1:400 dilution; Santa Cruz Biotechnology, Inc., Santa Cruz, CA, USA; p35/p25, C19, 1:100 dilution; Santa Cruz Biotechnology, Inc.) and overnight incubation at $4^{\circ} \mathrm{C}$. Secondary antibody horseradish peroxidase-labeled anti-rabbit or mouse $\operatorname{IgG}$ (1:1,000 dilution; Proteintech Group, Inc., Chicago, IL, USA) was added, the reaction was performed for $2 \mathrm{~h}$ and then developed with enhanced chemiluminiscence. $\beta$-actin was used as an internal reference. The experiment was repeated three times and gel image analysis was performed to determine the point density.

Changes in SOD activity and MDA content. Six mice from each group were anesthetized and decapitated and, each mouse brain was placed on an ice tray. Following rinsing with ice saline, the hippocampus was stripped and homogenized with $10 \%$ specimen-weight saline. The homogenate was then centrifuged at a low speed $(2,000 \mathrm{x} \mathrm{g})$ for $10 \mathrm{~min}$. The supernatant was placed inside a $-80^{\circ} \mathrm{C}$ refrigerator. A xanthine oxidase method was performed to test the SOD activity and a thiobarbituric acid (TBA) assay was performed to test the MDA content. SOD and MDA kits were purchased from the Nanjing Jiancheng Bioengineering Institute (Nanjing, Jiangsu, China).

Statistical analysis. Data are expressed as the mean \pm standard deviation and processed using SPSS 13.0 software. The single-factor analysis of variance was applied. An LSD test was used to compare the groups and $\mathrm{P}<0.05$ was considered to indicate a statistically significant difference.

\section{Results}

General observation. The majority of the mice in the MG demonstrated dull fur, depression and reduced activity. Mice in the TG also exhibited similar symptoms after 1 week, however the symptoms gradually disappeared after 1 month. These symptoms did not manifest in the mice in the SO group. Mice in all groups had no significant limb paralysis. The number of deaths in each treatment was as follows: five mice in the SO (mortality rate, $7.14 \%$ ), 30 mice in the MG (mortality rate, $33.33 \%$ ) and 28 mice in the TG (mortality rate, $31.1 \%$ ).

Comparison of learning and memory scores. The swimming time of all the mice in the MG on the 29th, 43rd and 57th day postoperative was significantly longer than that in the SO $(\mathrm{P}<0.05)$, whereas the swimming time of all the mice in the TG was significantly shorter than that in the MG $(\mathrm{P}<0.05)$. On the 30th, 44th and 58th day postoperative, the swimming time of all mice in the MG was significantly longer than that in the SO $(\mathrm{P}<0.05)$, whereas the swimming time of all the mice in the TG was significantly shorter than that in the MG $(\mathrm{P}<0.05)$. No significant difference was observed when the swimming time in the TG was compared with that in the SO group ( $\mathrm{P}>0.05)$. The learning and memory scores of each group are displayed in Fig. 1 and Fig. 2.

Pathological changes in the hippocampal CAl region. The pyramidal cells in the hippocampal CA1 region of SO mice were tightly packed and demonstrated a clear outline; the

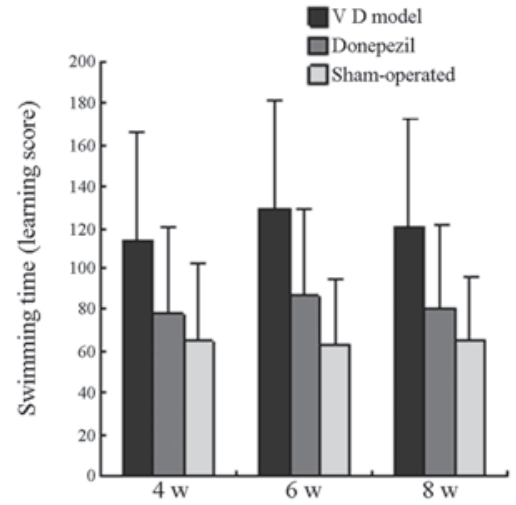

Figure 1. Comparison of swimming time in the water maze on the postoperative 4 th, 6 th and 8 th week of each group (learning score). w, week.

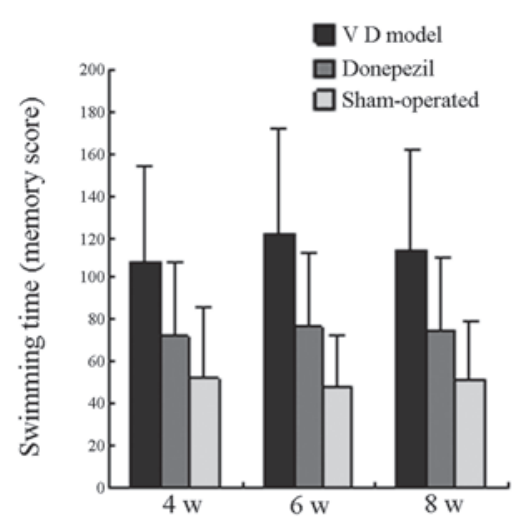

Figure 2. Comparison of swimming time in the water maze on the postoperative 4 th, 6th and 8 th week of each group (memory score). w, week.

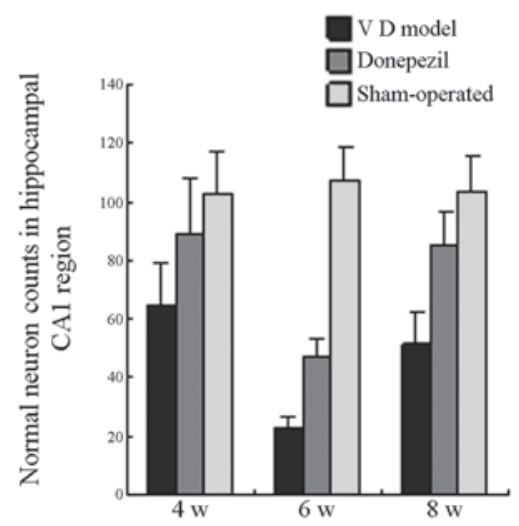

Figure 3. Comparison of normal neurons counts in hippocampal CA1 region on the postoperative 4 th, 6 th and 8 th week of each group.

nucleus was large and round with a clear nucleolus. The hippocampal pyramidal cell layers in the MG mice were few and loosely arranged. The nucleus reduced in size and was deeply stained, and the normal nerve cell count decreased. This decrease was most prominent following six weeks of surgery. Only a small number of cells exhibited a normal morphology. Compared with the normal neuron count, the neuron count in the MG mice was significantly reduced following 4 and 8 weeks postoperation $(\mathrm{P}<0.05)$. By contrast, the pyramidal cells in the TG mice were arranged neatly. The layers were 


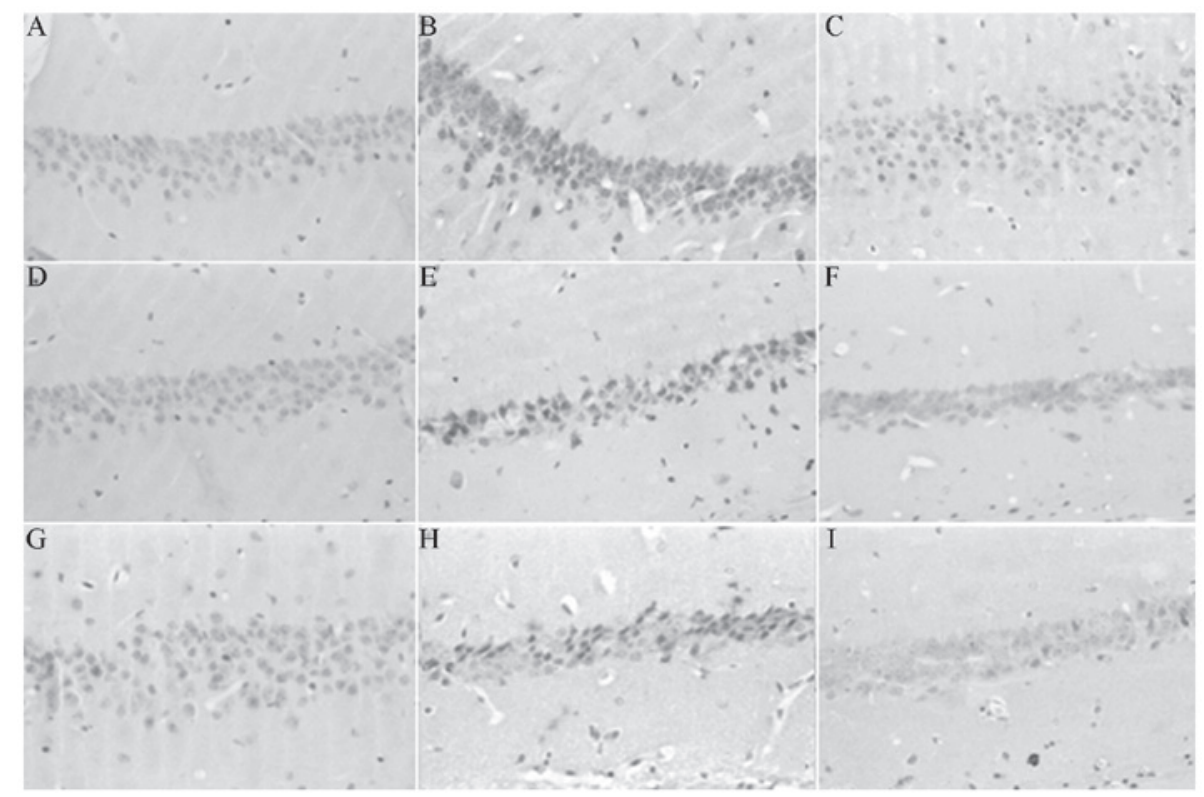

Figure 4. Expression of calpain I in the hippocampal CA1 region on the postoperative 4th, 6th and 8th week of each group (immunohistochemistry, $\mathrm{x} 400$ ). (A) SO on the postoperative 4th week; (B) MG on the postoperative 4th week; (C) TG on the postoperative 4th week; (D) SO on the postoperative 6th week; (E) MG on the postoperative 6th week; (F) TG on the postoperative 6th week; (G) SO on the postoperative 8th week; (H) MG on the postoperative 8th week; (I) TG on the postoperative 8th week. SO, sham operation group; TG, treatment group; MG, model group.

clear and free from nuclear condensation. The count of normal nerve cells was significantly higher than that in the MG mice $(\mathrm{P}<0.05)$. The comparison between the TG and SO mice did not demonstrate a significant difference on the 4 and 8 week postoperation ( $\mathrm{P}>0.05)$. As shown in Fig. 3, the neuron count in the TG mice remained significantly lower than that in the SO mice 6 weeks postoperation $(\mathrm{P}<0.05)$.

Calpain I expression in the hippocampal CAl region. In the $\mathrm{SO}$, the cellular layers in the hippocampal CA1 region on the 4,6 and 8th week postoperative were clear, with only a few traces of intracytoplasmically expressed calpain I. In the MG, the neuron structure in the hippocampal CA1 region on the 4 , 6 and 8th week postoperative was loose. The mean OD value of calpain I in neuronic intracytoplasm significantly increased $(\mathrm{P}<0.05)$ when compared with that in the SO. This increase was more apparent in the 6th week postoperative. As displayed in Fig. 4, the calpain I expression in the TG at the three time points was significantly lower than that in the MG $(\mathrm{P}<0.05)$ and close to that in the $\mathrm{SO}(\mathrm{P}>0.05)$.

CDK5 and p 25 protein expression. The CDK 5 and $\mathrm{p} 25$ protein expression in the hippocampal tissues of the MG mice on the 4, 6 and 8 th week postoperative of cerebral I/R were significantly higher than those in the $\mathrm{SO}(\mathrm{P}<0.05)$. This result suggests that $\mathrm{CDK} 5 / \mathrm{p} 25$ protein expression exhibited a sustained increase within eight weeks following cerebral I/R. The CDK 5 and p25 protein expression in the TG at the three time points significantly decreased when compared with those in the MG. The difference is statistically significant at $\mathrm{P}<0.05$ (Fig. 5 and Fig. 6).

SOD activity and MDA content. On the 4th, 6th and 8th week postoperative, the SOD activity in the MG was significantly higher than that in the SO $(\mathrm{P}<0.05)$, whereas SOD activity in the TG was significantly lower than that in the MG $(\mathrm{P}<0.05)$ and slightly higher than that in the SO with no significant difference $(\mathrm{P}>0.05)$. On the 4th, 6th and 8th week postoperative, the MDA activity in the MG was significantly higher than that in the SO group $(\mathrm{P}<0.05)$, whereas MDA activity in the TG was significantly lower than that in the MG $(\mathrm{P}<0.05)$. No significant difference was observed when MDA activity in the TG was compared with that in the SO group ( $\mathrm{P}>0.05$; Fig. 7).

\section{Discussion}

Previous studies demonstrated that ischemia is able to lead to neuronal cell death in vulnerable regions of the brain, particularly in the CA1 region of the hippocampus $(19,20)$. This finding is partly consistent with the results of the present study. By observing the histological morphology, it was demonstrated that the normal neuron count in the hippocampal CA1 region is significantly reduced within 8 weeks following cerebral $\mathrm{I} / \mathrm{R}$, along with deficits in the water maze performance. The findings of the present study support the theory that ischemic damage in the hippocampus causes a serious impairment of spatial memory tasks (21). The number of normal neurons in the MG on the 6th week postoperative was significantly lower than that in the SO and that in the MG on the 4 and 8th week postoperative. This result suggests that cerebral I/R injury exhibited a progressively increasing trend within 6 weeks. As time passed, the damage gradually reduced and the neuronal function began to recover. Consistent with the present study is one study that also highlights the inherent ability of the brain to form new nerve cells following ischemic damage (22). Overall, ischemic animals treated with $\mathrm{DH}$ performed better than those in the MG, suggesting that $\mathrm{DH}$ has a neuroprotective effect.

To explore the biochemical mechanisms that may be responsible for the observed histopathological and behavioral changes, the expression of calpain I and CDK5/p25 was 


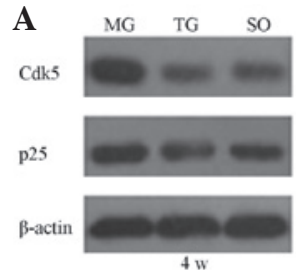

B

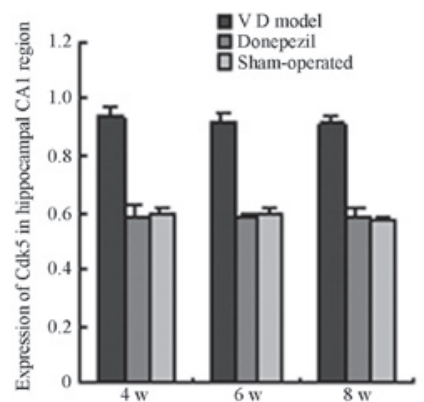

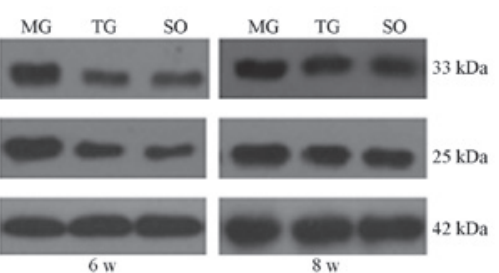

C

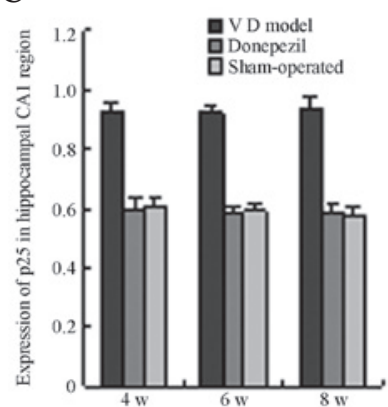

Figure 5. Expression of CDK5 and p25 in the hippocampus on the postoperative 4th, 6th and 8th week of each group. CDK5, cyclin-dependent kinase 5; $\mathrm{SO}$, sham operation group; TG, treatment group; MG, model group.

examined. CDK5/p25 regulates numerous signaling cascades and is thought to be an important mediator of learning and memory (23). A previous study revealed that the inhibition of CDK5/p25 activity provides a promising therapeutic avenue during or following stroke. This finding suggests that increases in CDK5/p25 activity leads to neuronal death (24). These data support the results of the present study, which demonstrated that calpain I and CDK5/p25 in the ischemic group were significantly increased in the hippocampus, particularly during the 6th week (postoperative) of ischemia. The increase in calpain I and CDK5/p25 in the ischemic group was accompanied by neuronal impairment in the hippocampal CA1 region and a decrease in learning and memory ability. The possible reasons were as follows: under the conditions of ischemia and hypoxia, $\mathrm{Ca}^{2+}$ overload was observed in hippocampal neurons, thereby activating calpain I (6) and consequently resulting in the increase in p25 generation. Therefore, CDK 5/p25 activity was maladjusted, thus leading to the inability of CDK5 to perform normal physiological functions toward synapse-related substances. These conditions affect long term potentiation generation and cause a decrease in learning and memory ability (7).

Oxidative stress is important in the pathogenesis of $\mathrm{I} / \mathrm{R}$ injury (2). The process is accompanied by elevated free radicals. These radicals initiate a radical chain reaction of signaling pathways that involve the mitochondria and lead to cell death (25). The oxidation and antioxidation status following cerebral ischemia in mice is determined by the activities of lipid peroxides and SOD and by the levels of catalase and glutathione peroxidase. MDA is used as a marker of lipid peroxidation and its content may reflect the level of lipid peroxide (26).

The present study explored SOD activity and MDA content in brain tissue and demonstrated that MDA content was significantly higher in the hippocampus following 8 weeks of cerebral I/R; an increase in SOD activity was also observed. As SOD may function as an antioxidant enzyme in

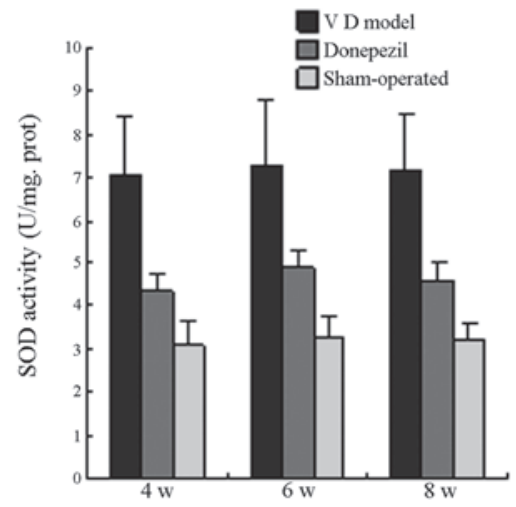

Figure 6. Changes in SOD activity in the hippocampus on the postoperative 4th, 6th and 8th week of each group. SOD, superoxide dismutase.

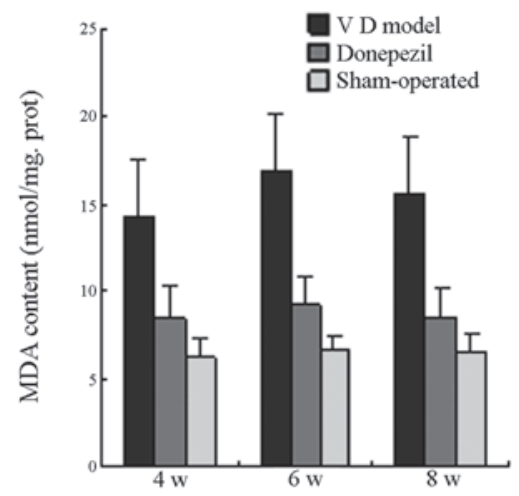

Figure 7. Changes in MDA content in the hippocampus on the postoperative 4 th, 6th and 8th week of each group. MDA, malondialdehyde.

ischemia-induced neuronal damage, increased SOD activity found in this study may be explained by a compensatory increase in antioxidant activity that targeted the increase in free radical production associated with the brain antioxidant mechanism, which was activated under serious oxidative stress (27). The study also confirmed that DH is able to reduce SOD activity and MDA content. These findings suggest that $\mathrm{DH}$ has a protective function in cerebral I/R-induced memory impairment.

Growing evidence suggests that increased ROS production and cytosolic free $\mathrm{Ca}^{2+}$ overload, either independently or co-operatively, are major contributors to I/R-induced injury $(2,3,5)$. Our findings are consistent with this evidence. In the present study, the expression of CDK5/p25 in the hippocampal CA1 region was demonstrated to increase following cerebral $\mathrm{I} / \mathrm{R}$, along with the increase in SOD activity and MDA content. Peroxiredoxin $2(\operatorname{Pr} x 2)$ is an antioxidative enzyme with peroxidase activity (28). A previous study demonstrated that Prx2 is a critical cytoplasmic target of Cdk5. In the cerebral ischemia model, an abnormal increase in the expression of CDK5 in the hippocampal CA1 region, accompanied by increasing Prx 2 phosphorylation, was observed. This finding suggests that CDK5 may affect ROS generation by affecting Prx2 (29). The involvement of this mechanism in the pathogenesis of cerebral I/R requires further exploration.

In the mouse model of the present study, DH was demonstrated to be capable of ameliorating learning and memory 
function through the calpain I/CDK5 signaling pathway in the hippocampus. DH is now in clinical trials for AD treatment, however emerging information suggests a potential role of $\mathrm{DH}$ in the therapy of ischemic disorders (20). The pretreatment with DH may reduce the release of lactic dehydrogenase, thus suggesting that anti-ischemic brain damage as a consequence of DH is not associated with the inhibition of cholinesterase (17). DH may inhibit the fast inflow of $\mathrm{Na}^{+}$and $\mathrm{Ca}^{2+}$, reduce the release of glutamate and therefore oppose the neurotoxicity induced by depolarization (30). The inhibition of $\mathrm{Ca}^{2+}$ influx by DH is likely to be the cause of the decreased expression of calpain I and CDK5/p25, and improved memory function in the present study. In addition, the neuroprotective effects of DH may be caused by ROS reduction by SOD and MDA. These results suggest that antioxidation may be one of the key mechanisms of neuroprotection following DH treatment.

This study is not without its limitations. Firstly, further study is needed to investigate which CDK5/p25 signaling pathway regulates oxidative damage. Secondly, excitotoxicity, inflammation and apoptosis are also involved in cerebral I/R injury $(31,32)$. Whether these mechanisms, except $\mathrm{Ca}^{2+}$ overload and antioxidant effects, are responsible for the neuroprotection of $\mathrm{DH}$ needs to be explored further.

In conclusion, the present study demonstrated that $\mathrm{DH}$ has a significant protective effect on cerebral I/R-induced brain injury. Inhibition of $\mathrm{Ca}^{2+}$ overload and antioxidant ability appears to be the basic and important mechanisms of the neuroprotective effect of $\mathrm{DH}$. These results suggest that DH may be a promising therapeutic drug for the treatment of cognitive impairment following cerebral I/R damage.

\section{Acknowledgements}

This study was supported by the National Natural Science Foundation of China (grant no. 81241037).

\section{References}

1. Yan BC, Park JH, Ahn JH, Lee JC, Won MH and Kang IJ: Postsynaptic density protein (PSD)-95 expression is markedly decreased in the hippocampal CA1 region after experimental ischemia-reperfusion injury. J Neurol Sci 330: 111-116, 2013.

2. Tu Q, Wang R, Ding B, Zhong W and Cao H: Protective and antioxidant effect of Danshen polysaccharides on cerebral ischemia/reperfusion injury in rats. Int J Biol Macromol 60: 268-271, 2013.

3. Wang Q, Kalogeris TJ, Wang M, Jones AW and Korthuis RJ: Antecedent ethanol attenuates cerebral ischemia/reperfusion-induced leukocyte-endothelial adhesive interactions and delayed neuronal death: role of large conductance, $\mathrm{Ca}^{2+}$-activated $\mathrm{K}^{+}$channels. Microcirculation 17: 427-438, 2010.

4. Zhang F, Guo A, Liu C, Comb M and Hu B: Phosphorylation and assembly of glutamate receptors after brain ischemia. Stroke 44: 170-176, 2013

5. Racay P, Tatarkova Z, Chomova M, Hatok J, Kaplan P and Dobrota D: Mitochondrial calcium transport and mitochondrial dysfunction after global brain ischemia in rat hippocampus. Neurochem Res 34: 1469-1478, 2009.

6. Peng S, Kuang Z, Zhang Y, Xu H and Cheng Q: The protective effects and potential mechanism of Calpain inhibitor Calpeptin against focal cerebral ischemia-reperfusion injury in rats. Mol Biol Rep 38: 905-912, 2011.

7. Su SC and Tsai LH: Cyclin-dependent kinases in brain development and disease. Annu Rev Cell Dev Biol 27: 465-491, 2011.

8. Chen J and Wang ZF: Roles of cyclin-dependent kinase 5 in central nervous system development and neurodegenerative diseases. Sheng Li Xue Bao 62: 295-308, 2010.
9. Ghosh A, Sarkar S, Mandal AK and Das N: Neuroprotective role of nanoencapsulated quercetin in combating ischemia-reperfusion induced neuronal damage in young and aged rats. PLoS One 8: e57735, 2013.

10. Harry GJ and Lefebvre d'Hellencourt C: Dentate gyrus: alterations that occur with hippocampal injury. Neurotoxicology 24: 343-356, 2003.

11. Knapp LT and Klann E: Role of reactive oxygen species in hippocampal long-term potentiation: contributory or inhibitory? J Neurosci Res 70: 1-7, 2002.

12. Kishida KT and Klann E: Sources and targets of reactive oxygen species in synaptic plasticity and memory. Antioxid Redox Signal 9: 233-244, 2007.

13. Zhou L, Aon MA, Liu T and O'Rourke B: Dynamic modulation of $\mathrm{Ca}^{2+}$ sparks by mitochondrial oscillations in isolated guinea pig cardiomyocytes under oxidative stress. J Mol Cell Cardiol 51: 632-639, 2011.

14. Winblad B, Engedal K, Soininen H, et al: A 1-year, randomized, placebo-controlled study of donepezil in patients with mild to moderate AD. Neurology 57: 489-495, 2001.

15. Fujiki M, Kobayashi H, Uchida S, Inoue R and Ishii K: Neuroprotective effect of donepezil, a nicotinic acetylcholine-receptor activator, on cerebral infarction in rats. Brain Res 1043: 236-241, 2005.

16. Takada Y, Yonezawa A, Kume T, et al: Nicotinic acetylcholine receptor-mediated neuroprotection by donepezil against glutamate neurotoxicity in rat cortical neurons. J Pharmacol Exp Ther 306: 772-777, 2003.

17. Akasofu S, Kimura M, Kosasa T, Sawada K and Ogura H: Study of neuroprotection of donepezil, a therapy for Alzheimer's disease. Chem Biol Interact 175: 222-226, 2008.

18. Akasofu S, Sawada K, Kosasa T, Hihara H, Ogura H and Akaike A: Donepezil attenuates excitotoxic damage induced by membrane depolarization of cortical neurons exposed to veratridine. Eur J Pharmacol 588: 189-197, 2008.

19. Fang S, Yan B, Wang D, et al: Chronic effects of venlafaxine on synaptophysin and neuronal cell adhesion molecule in the hippocampus of cerebral ischemic mice. Biochem Cell Biol 88: 655-663, 2010.

20. Min D, Mao X, Wu K, et al: Donepezil attenuates hippocampal neuronal damage and cognitive deficits after global cerebral ischemia in gerbils. Neurosci Lett 510: 29-33, 2012.

21. Block F: Global ischemia and behavioural deficits. Prog Neurobiol 58: 279-295, 1999.

22. von Euler M, Bendel O, Bueters T, Sandin J and von Euler G: Profound but transient deficits in learning and memory after global ischemia using a novel water maze test. Behav Brain Res 166: 204-210, 2006.

23. Shukla V, Skuntz S and Pant HC: Deregulated Cdk5 activity is involved in inducing Alzheimer's disease. Arch Med Res 43: 655-662, 2012.

24. Mitsios N, Pennucci R, Krupinski J, et al: Expression of cyclindependent kinase 5 mRNA and protein in the human brain following acute ischemic stroke. Brain Pathol 17: 11-23, 2007.

25. Christophe M and Nicolas S: Mitochondria: a target for neuroprotective interventions in cerebral ischemia-reperfusion. Curr Pharm Des 12: 739-757, 2006.

26. Guo C, Tong L, Xi M, Yang H, Dong $\mathrm{H}$ and Wen A: Neuroprotective effect of calycosin on cerebral ischemia and reperfusion injury in rats. J Ethnopharmacol 144: 768-774, 2012.

27. Liu C, Wu JL, Gu J, et al: Baicalein improves cognitive deficits induced by chronic cerebral hypoperfusion in rats. Pharmacol Biochem Behav 86: 423-430, 2007.

28. Rhee SG, Yang KS, Kang SW, Woo HA and Chang TS: Controlled elimination of intracellular $\mathrm{H}(2) \mathrm{O}(2)$ : Regulation of peroxiredoxin, catalase, and glutathione peroxidase via post-translational modification. Antioxid Redox Signal 7: 619-626, 2005.

29. Rashidian J, Rousseaux MW, Venderova K, et al: Essential role of cytoplasmic cdk5 and Prx2 in multiple ischemic injury models, in vivo. J Neurosci 29: 12497-12505, 2009.

30. Bendel O, Prunell G, Stenqvist A, et al: Experimental subarachnoid hemorrhage induces changes in the levels of hippocampal NMDA receptor subunit mRNA. Brain Res Mol Brain Res 137: 119-125, 2005.

31. Koh PO: Ischemic injury decreases parvalbumin expression in a middle cerebral artery occlusion animal model and glutamate-exposed HT22 cells. Neurosci Lett 512: 17-21, 2012.

32. Kliper E, Bashat DB, Bornstein NM, et al: Cognitive decline after stroke: relation to inflammatory biomarkers and hippocampal volume. Stroke 44: 1433-1435, 2013. 